Meta

Journal des traducteurs

Translators' Journal

\title{
Traduire la Bible et le Coran à Jérusalem : André Chouraqui
}

\section{Francine Kaufmann}

Volume 43, numéro 1, mars 1998

La traduction et l'interprétation en Israël

Translation and Interpreting in Israel

URI : https://id.erudit.org/iderudit/003294ar

DOI : https://doi.org/10.7202/003294ar

Aller au sommaire du numéro

Éditeur(s)

Les Presses de l'Université de Montréal

ISSN

0026-0452 (imprimé)

1492-1421 (numérique)

Découvrir la revue

Citer cet article

Kaufmann, F. (1998). Traduire la Bible et le Coran à Jérusalem : André Chouraqui. Meta, 43(1), 142-156. https://doi.org/10.7202/003294ar

\section{Résumé de l'article}

Traducteur des trois textes fondamentaux du judaïsme, du christianisme et de l'Islam, André Chouraqui a commencé son entreprise avant même de s'installer à Jérusalem (en 1958). Mais son enracinement quotidien dans le paysage et la langue de la Bible ont favorisé un regard nouveau sur des textes devenus un héritage familier des peuples du Livre, mais trop souvent déchiffrés à coup de dictionnaire. Cet article entreprend de retracer l'itinéraire qui a mené l'enfant du Maghreb à s'approprier la culture française, à se réapproprier la culture hébraïque. Il propose aussi des pistes et des références bibliographiques invitant les traductologues à découvrir l'œuvre d'un traducteur biblique trop souvent appréhendé sous le seul angle confessionnel (voire politique) ou analysé à la lumière des clichés de la polémique entre "ciblistes" et "sourciers". 


\title{
TRADUIRE LA BIBLE ET LE CORAN À JERRUSALEM : ANDRE CHOURAQUI
}

\author{
FRANCINE KAUFMANN \\ Université Bar-Ilan, Ramat-Gan, Israël
}

\begin{abstract}
Résumé
Traducteur des trois textes fondamentaux du judaïsme, du christianisme et de l'Islam, André Chouraqui a commencé son entreprise avant même de s'installer à Jérusalem (en 1958). Mais son enracinement quotidien dans le paysage et la langue de la Bible ont favorisé un regard nouveau sur des textes devenus un héritage familier des peuples du Livre, mais trop souvent déchiffrés à coup de dictionnaire. Cet article entreprend de retracer l'itinéraire qui a mené l'enfant du Maghreb à s'approprier la culture française, à se réapproprier la culture hébrä̈que. Il propose aussi des pistes et des références bibliographiques invitant les traductologues à découvrir l'œuvre d'un traducteur biblique trop souvent appréhendé sous le seul angle confessionnel (voire politique) ou analysé à la lumière des clichés de la polémique entre «ciblistes» et «sourciers».
\end{abstract}

\begin{abstract}
Translator of three fundamental texts on Judaism, Christianity and Islam, André Chouraqui became involved in this undertaking even before settling in Jerusalem (in 1958). His everyday association with the land and language of the Bible have enabled him to view these familiar, but all too frequently translated word-for-word texts from a fresh perspective. This article shows how a child of the Maghreb became integrated into the French culture and subsequently reintegrated into Hebrew culture. It provides bibliographical references to invite translation specialists to discover the work of a biblical translator too often regarded solely from a confessional viewpoint or analyzed in terms of target-text versus source-text based.
\end{abstract}

\section{PRÉAMBULE : JÉRUSALEM ET LA PAROLE DIVINE}

Si l'on aime les symboles, on trouvera naturel qu'André Chouraqui, l'homme qui a passé vingt ans de sa vie à traduire la Bible juive et chrétienne ainsi que le Coran, habite la ville trois fois sainte de Jérusalem, dans le quartier d'Abou Tor, à une douzaine de kilomètres à vol d'oiseau de Bethléem où vécut, presque en reclus, saint Jérôme (v. 331-v. 420), patron des traducteurs, auteur de la Vulgate, version latine de la Bible hébraïque (hebraïca veritas). En direction opposée, sous ses fenêtres et de l'autre côté de la vallée de la Géhenne et de la Citadelle de David, s'élève le Mont du Temple (appelé aussi Esplanade des Mosquées). C'est là que Mahommet aurait fait son ascension au ciel, non loin de la via Dolorosa, dans un périmètre qui aurait vu, près de dix siècles auparavant, soixante-dix érudits (certains disent soixante-douze) choisis par le Grand Prêtre du Temple de Jérusalem partir pour Alexandrie afin de traduire en grec la Bible hébraïque pour la Bibliothèque du roi d'Égypte Ptolémée (sans doute Ptolémée II, 308-246 avant Jésus-Christ). La traduction juive des Sages de Jérusalem, les Septante (au troisième siècle avant notre ère) est l'un des plus anciens corpus littéraires connus. En tout cas, la légende et l'histoire se mêlent pour accorder à Jérusalem une place de choix dans l'histoire de la traduction sacrée, et ce n'est pas par hasard que les éditions du Cerf, pourtant situées à Paris, ont donné à leur bible devenue classique (et traduite aujourd'hui dans d'autres langues) le nom prestigieux de 
Bible de Jérusalem, réalisée, il est vrai, dans l'esprit et sous la direction de l'École biblique et archéologique française de Jérusalem (fondée en 1890 et sise rue Naplouse, à Jérusalem Est).

C'est encore à Jérusalem que vit et travaille le rabbin Adin Steinsaltz, qui, depuis 1966, a entrepris de traduire en hébreu israélien le Talmud (développement rabbinique de la Bible et tradition orale, couché par écrit entre le second et le sixième siècle). Le rabbin Steinsaltz vocalise et annote la Michna (hébraïque), traduisant de l'araméen et expliquant la Guemara. En 1996, il célébrait le trentième anniversaire de son entreprise (et le trentième volume traduit et commenté du Talmud). Mais depuis, dans l'Institut israélien des publications talmudiques qu'il a créé à Jérusalem et qu'il dirige (non loin de la Porte de Jaffa), le Talmud Steinsaltz est en cours de traduction en anglais, en français et en russe (selon une méthode qui aurait mérité à elle seule un long article dans ce numéro spécial de Meta).

Quoi qu'il en soit, André Chouraqui, traducteur de la Bible avant même son installation en Israël, fut longtemps un traducteur sagement classique, aux phrases élégantes et soigneusement coulées dans le moule de la langue française. Sa traduction du moraliste juif médiéval Bahya Ibn Paquda, dont Les devoirs des cœurs sont truffés de versets bibliques (1950), puis du Cantique des Cantiques (dès 1951) et des Psaumes (1956) font dire à son maître, l'universitaire Georges Vajda, que ses traductions sont plus «littéraires» que «philologiques» (La Revue critique, mars 1951), et le frère Michel Mabourdette, parlant de la traduction de Bahya, conclut en 1952 :

M. Chouraqui a le rare mérite de nous présenter un texte non seulement français mais beau, écrit dans une langue sobre, pleine, qui s'harmonise admirablement avec l'enseignement donné et sans aucune enflure, s'ouvre périodiquement au grand souffle de la poésie biblique. (La Revue thomiste)

Même Henri Meschonnic (1970), qui critique «le calque inviable, le français fictif d'Edmond Fleg quand il traduisait Le livre du Commencement» qualifie de «livresque-poétisant-médiévisant, le dialecte littéraire de Chouraqui dans son Cantique des Cantiques» dont David Jassine (1971) admire «le français plein d'élégance».

Comment donc expliquer l'évolution et le parti pris qui pousse André Chouraqui (auteur de plusieurs recueils de poèmes) à abandonner le point de vue «cibliste» (privilégiant la langue d'accueil et la «lisibilité»), pour adopter un point de vue «sourcier», une entreprise de «décentrement» accentuant l'«étrangeté» de la civilisation biblique (pour reprendre des concepts chers à Jean-René Ladmiral, 1986) ? L'image qu'ont les lecteurs de la «Bible Chouraqui» n'est-elle pas celle d'un texte rocailleux, abrupt, aux beautés sauvages et ardues, parcouru (sans doute pour cela même) par un souffle vivifiant qui rend à la Bible sa fraîcheur et l'actualise, mais qui fait violence à la langue française ?

À lire les nombreuses interviews accordées par André Chouraqui dans la presse générale ou confessionnelle, les raisons de ses nouveaux choix sont nombreuses. Il s'agissait pour lui, avant tout, de secouer des siècles d'habitudes traductionnelles qui ont enfoui la parole de Dieu dans des expressions figées, souvent éloignées des significations de l'original, dénaturées, édulcorées, simplifiées par le désir de rendre clair et rationnel ce qui était touffu et obscur dans l'hébreu, mais consacrées par l'usage. Chouraqui souhaitait redonner vie à la langue et aux images employées dans la Bible, comme il voyait, sous ses yeux, l'hébreu et la civilisation juive héritée de l'hébraïsme ancien reprendre vie dans la Jérusalem où il s'était installé. Car, à n'en pas douter, c'est la rencontre quotidienne avec les paysages et avec le langage de la Bible qui a modifié sa sensibilité et lui a fait ressentir l'incongruité et l'inadéquation des formules adoptées par convention et par tradition. Cessant d'être fossilisée dans les dictionnaires bibliques des séminaires, voilà que la langue de 
la Bible se mettait à résonner dans la bouche de ses cinq enfants (dont l'hébreu est la langue maternelle), à la radio, dans la rue, aux portes du désert commençant sous les fenêtres de sa maison d'où l'on voit passer les bergers et fleurir les plantes évoquées dans la Bible. Et lorsqu'on s'étonne qu'il ait pu, seul, réaliser une nouvelle traduction de la Bible alors que l'équipe des traducteurs de la T.O.B. (la Traduction Ecuménique de la Bible, en français) compte une centaine de spécialistes, Chouraqui répond :

Jamais je n'aurais pu conduire ainsi mon travail si l'hébreu n'était pas devenu ma langue [...] Quand je relis mes traductions du Cantique des Cantiques et des Psaumes, de 1950 à 1956, elles me semblent caduques. Mon regard était flou. J'ai repris tout cela; de façon plus précise. Quand un mot compte 80 ou 100 sens différents, comment savoir, de façon livresque, lequel est le bon ? Je ne traduis plus au vu du dictionnaire, mais en sachant, de l'intérieur, de quoi il s'agit. C'était lointain; et tout d'un coup, c'est devenu réel. (Réforme, 26 janvier 1974)

Henri Meschonnic, «sourcier» lui aussi, à bien des égards, se refuse à accepter ces explications. Pour lui, l'entreprise de Chouraqui est un vaste coup médiatique pour promouvoir :

une régression linguistique, un faux poétique et une trahison du juif [...] Puisque c'est la première traduction d'un hébréophone, «homme de Jérusalem», on veut dire par là qu'il connaît l'hébreu. Mais cet argument publicitaire n'a aucune valeur linguistique, car il confond le bilinguisme et la traduction [...]. L'amplification de la presse en vient même à lier la traduction de Chouraqui à la résurrection de l'hébreu, celle-ci étant une «raison exceptionnelle et irrécusable de celle-là». Mais «parler quotidiennement l'hébreu» n'aidera pas plus à traduire le grec du Nouveau Testament que l'hébreu biblique, car traduire ne saurait se ramener au seul problème de la connaissance d'une langue de départ. (La Quinzaine littéraire, $1^{\mathrm{er}}$ au 15 septembre 1974)

Et Meschonnic de conclure que le résultat d'ensemble est «juxtalinéaire», «illisible» et «incompréhensible». "Il ne suffit pas d'être "homme de Jérusalem" mais il faut être aussi poète français» (ibid). Désormais, Meschonnic ne manque jamais de démontrer que Chouraqui entend se placer dans une filière littéraliste, une «réaction surtout juive», depuis «Alexandre Weil en 1890, jusqu'à l'aquiléisme des traductions d'Edmond Fleg» (1981 : 32). Ainsi, affirme-t-il, «l'hébraïsation, la défrancisation se font par un calque étymologique, lexical, syntaxique» (ibid: 33), produisant une «Bible en décalcomanie» (1978).

J'ai tenté de montrer ailleurs (Kaufmann, 1990, 1997, à paraître) les dimensions complexes de l'approche juive, taxée de littéraliste par ceux qui projettent sur une certaine traduction juive des stéréotypes éculés issus de l'antique polémique théologique sur une soi-disant opposition entre le respect de la lettre (par les juifs) et le respect de l'esprit (par la tradition chrétienne). Je voudrais ici fournir une sorte de monographie de Chouraqui qui, par des éléments biographiques et surtout bibliographiques (cf. les références en fin d'article), permettront de mieux apprécier sa démarche. J'espère contribuer par là à faire sortir André Chouraqui du seul cercle des amitiés judéo-chrétiennes et judéo-arabes, où il est bien connu, pour le faire mieux prendre en compte par les traductologues (qui l'abordent essentiellement à travers les lunettes déformantes de la polémique avec Meschonnic, bien qu'aujourd'hui des études «littéraires» ou «traductologiques» commencent à paraître (cf. Gergely, 1980; de Vries et Verheij, 1997; Kaufmann, 1997; Aslanov, à paraître).

Par ailleurs, une approche biographique révélera les sources de son intérêt et de ses compétences pour les langues et les trois religions du livre et explique comment la traduction des textes sacrés est devenu, chez cet homme à cheval entre trois cultures, une mission de paix et d'invitation à la coexistence. 


\section{LES ORIGINES}

Né le 11 août 1917, à Aïn Témouchent, non loin d'Oran, à l'ouest de l'Algérie, André Chouraqui s'est installé à Jérusalem en 1958, au milieu chronologique de sa vie. Dans cette ville dont il a été l'adjoint au maire (de 1965 à 1973), il a traduit non seulement la Bible hébraïque, mais aussi les Évangiles et le Coran.

Homme de «l'Orient», ayant grandi dans une famille juive arabophone dont les ancêtres parlaient l'arabe depuis près d'un millénaire, familier des sonorités hébraïques entendues à la synagogue et au Talmud Thora dès l'âge de trois ans (et répétées de mémoire, sans en comprendre encore les significations), éduqué dans l'Algérie française du décret Crémieux (qui, d'un trait de plume, avait transformé les Juifs autochtones en citoyens français bon teint, le 24 octobre 1870), André Chouraqui acquit ses premiers rudiments de français au jardin d'enfants (catholique) puis à l'école primaire (laïque).

Dans son autobiographie, L'amour fort comme la mort (éditions Robert Laffont, 1990), André Chouraqui se souvient que la vie des juifs était rythmée par les cycles liturgiques qui les transformaient en contemporains d'Abraham et de la sortie d'Égypte, le regard tourné vers Jérusalem où devait les ramener la fin de l'exil : «Nous vivions une aventure transhistorique» (p. 59).

En attendant, nous étions absents au monde dans lequel notre exil nous enfermait. Nous naissions, nous vivions, et nous mourions dans les étroites limites de nos ghettos. Nous pouvions nouer des relations d'affaires avec les musulmans ou les chrétiens de notre entourage, nouer quelquefois de vraies amitiés; il était plus rare, jusqu'à une époque tardive, d'avoir avec eux de vrais échanges intellectuels. (pp. 58-59)

Qu'est-ce donc qui devait préparer le petit André-Natân à un rapprochement (caractérisé par un respect et un intérêt profond) avec les cultures de ses voisins sans jamais remettre en cause la sincérité de ses attaches juives et la solidarité avec le destin de son propre peuple ? Sans doute une insatiable curiosité intellectuelle mais aussi une éducation ouverte et les hasards d'une vie agitée.

Neuvième enfant d'une famille de dix (dont six seulement devaient survivre), André appartient à la bourgeoisie aisée d'Aïn Témouchent. Son père, Isaac Chouraqui, est négociant en céréales, membre du conseil municipal et président du consistoire israélite de la ville. De stricte observance pour lui-même mais très libéral pour les autres, il trouve naturel d'envoyer ses enfants dans des institutions chrétiennes ou laïques pourvu qu'elles puissent leur procurer la meilleure éducation. Ses ancêtres remontent aux exilés chassés d'Espagne par les persécutions et installés à Tlemcen, «la perle du Maghreb», dès la fin du XIV ${ }^{\mathrm{e}}$ siècle.

La rupture avec le monde protégé de l'enfance est consommée à l'âge de onze ans, lorsque le petit André (devenu boiteux et d'une sensibilité exacerbée après avoir contracté la poliomyélite) est envoyé au lycée d'Oran pour y poursuivre des études secondaires et conquérir le fameux baccalauréat. Sept ans d'internat, dans une discipline toute spartiate, détachent radicalement le petit Juif oriental de ses racines. Au lycée d'Oran, l'éducation est française, républicaine et laïque :

Nous étions non seulement déjudaïsés ou désislamisés, mais par surcroît athéisés. Nous étions livrés corps et âme non seulement aux mânes de Vercingétorix, mais plus gravement à ceux de Voltaire et des Encyclopédistes [...] C'est ainsi que je changeais de peuple élu, insidieusement et comme sans m'en apercevoir. (pp. 82-83)

Sa Bar-Mitsva, célébrée dans la grande synagogue d'Aïn Témouchent, en été 1929, marque son dernier lien formel avec les traditions juives. Il continue à jeûner à Kippour et à ne pas manger de pain à Pessa'h. Mais pour le reste, il devient un parfait petit Français. Il 
conserve quelques liens avec l'Orient grâce aux cours de langue et de grammaire arabes dispensés au lycée d'Oran. Mais il se passionne pour la littérature occidentale et lit durant les vacances d'été tout ce qui lui tombe sous la main : romanciers, poètes, appréciant particulièrement les romantiques. Quant à Dieu, il est relégué au magasin des accessoires.

\section{LA QUÊTE DE L'IDENTITÉ ET DE LA SPIRITUALITÉ}

Le premier contact adulte avec la Bible de ses ancêtres survient par des voies détournées... et chrétiennes. Entre ses deux baccalauréats, durant l'été 1934, André Chouraqui part en métropole pour subir une opération de la cheville qui le guérit partiellement de son infirmité. La clinique qui l'accueille, à Courbevoie, est tenue par une œuvre protestante. Deux jeunes infirmières lui font lire la Bible (dans la traduction protestante de Louis Segond) et s'engagent avec lui dans de longs débats (puis dans une correspondance) sur Dieu et les réalités spirituelles. Parallèlement, c'est la découverte des grands philosophes, des écrivains contemporains, de la musique classique et des balades à vélo. Le judaïsme est loin. Le jour de ses dix-huit ans, son baccalauréat en poche, ses parents décident qu'André quittera l'Algérie pour la France. C'est dit ! Il sera avocat.

Novembre 1935 : c'est la rentrée à la faculté de droit de Paris. Parallèlement, le futur traducteur des Évangiles reprend son dialogue avec Yvonne (la plus âgée des deux infirmières protestantes) et prend aussi l'habitude d'accompagner l'un de ses professeurs de droit à la messe du dimanche pour écouter l'organiste de Notre-Dame. Son athéisme est profondément ébranlé en même temps qu'il découvre une civilisation dont il n'avait, au mieux, qu'une connaissance livresque. «Les cathédrales, les églises, la musique religieuse, l'art chrétien, les couvents et les monastères [...] Quel choc pour le petit barbare que j'étais au sortir de mes déserts coloniaux» (ibid : 126).

Convaincu définitivement (après une «illumination» dans les Hautes-Alpes, en février 1937) que «Dieu existe», conscient que la Bible qu'il lit et médite en traduction française est bien éloignée du texte original hébraïque, André décide de se mettre à l'étude de son patrimoine.

J'étais juif et je ne savais pas clairement ce que cela pouvait bien vouloir dire. Que Hitler veuille me persécuter et, éventuellement me tuer pour cette raison, c'était là un fait évident qu'il n'était pas en mon pouvoir de modifier. Ce que du moins je pouvais faire, c'était de savoir le pourquoi de cette affaire qui me menaçait dans mon existence. (p. 157)

À Paris, un condisciple du lycée d'Oran, André Zaoui, le met en contact avec l'École rabbinique de France et lui donne ses premières leçons d'hébreu. Sans jamais vouloir devenir rabbin, André Chouraqui poursuit désormais des études parallèles de droit et de judaïsme, à Paris puis dans la clandestinité. De 1937 à 1939, il obtient sa licence puis son diplôme d'études supérieures de droit et poursuit son apprentissage de l'hébreu, de la Bible juive et de ses commentaires (avec Georges Vajda), du Talmud et de l'araméen (avec Abraham Back), tant à l'École rabbinique qu'à la Sorbonne et à l'École des hautes études. Il passe tous ses étés en Algérie mais revient chaque automne en France, même lorsque la guerre éclate et qu'il suit l'école rabbinique repliée d'abord à Vichy puis à ClermondFerrand.

Mais son «retour au judaïsme» (un judaïsme plus proche de celui des Hébreux et des mystiques médiévaux que de la tradition talmudique, une religiosité où la pratique religieuse est réduite au strict minimum) ne consomme pas la rupture avec les autres civilisations monothéistes. L'amour successif de deux jeunes catholiques (1938-1939) lui fait pénétrer plus avant la spiritualité exigeante de la foi, de la doctrine et des dogmes chrétiens. Puis, au début de 1940, lors d'un séjour de plusieurs mois dans le Sahara, il donne 
des cours de français au cadi (le juge musulman) en échange de leçons de Coran et d'arabe (qui lui seront utiles cinq ans plus tard, lorsqu'il passera, devant la faculté d'Alger, un diplôme supérieur de droit musulman et de coutumes indigènes). Dans une autre oasis, à Ghardaïa, il voit vivre, dans la tension mais côte à côte, une communauté juive de trois mille âmes qui lui fait pressentir ce qu'a pu être la vie quotidienne des juifs de l'Orient biblique, à l'époque de la Michna, les Kharéjites (une secte musulmane) et les pères blancs.

Revenu en France en mai 1940, il doit fuir Paris un mois plus tard. Il connaît les bombardements allemands à Orléans, repart pour l'Algérie où il se marie, s'inscrit au barreau d'Oran et commence un stage chez un bâtonnier. À la suite de la législation antijuive, de l'abrogation du décret Crémieux, et avant même la promulgation par Vichy du Statut des Juifs, le 20 octobre 1941, excluant les Juifs de la plupart des professions, André prend les devants en démissionnant, en juin 1941, tandis que ses parents sont ruinés.

C'est à cette époque que, avec l'aide du rabbin Isaac Rouche, adjoint du grand rabbin d'Oran, André Chouraqui entreprend de traduire Les devoirs des coeurs, un traité en arabe de l'un des plus grands penseurs juifs espagnols du XI $\mathrm{XI}^{\mathrm{e}}$ siècle, Bahya Ibn Paquda. Fourmillant de versets bibliques, cette œuvre constituera la première confrontation de Chouraqui avec les difficultés de la traduction biblique. Devant le petit groupe d'intellectuels juifs d'Oran jetés à la rue par les lois de Vichy, il prononce sa première conférence publique sur le thème «Comment lire la Bible ?». Il a vingt-quatre ans. Il constate aujourd'hui que la problématique et la plupart des thèmes de ses futures traductions bibliques se trouvent déjà en germe dans cette conférence.

Il rejoint bientôt l'École rabbinique repliée près de Clermont-Ferrand où il entame sa quatrième année d'étude. Il poursuit sa traduction de Bahya tout en approfondissant, sa connaissance de la théologie et de la mystique des juifs, des chrétiens et des musulmans (Maïmonide, saint Thomas d'Aquin et les commentateurs du Coran). Mais la guerre se rapproche. En juillet 1942, après la rafle et la déportation des Juifs et des Alsaciens de l'Université de Strasbourg repliée à Clermont-Ferrand, les Juifs sont expulsés de la ville... L'École rabbinique est démantelée et décimée.

Durant plus de deux ans, André Chouraqui va vivre dans la clandestinité. Il prend contact avec les réseaux naissants de la résistance juive. Il sillonne les villages pour cacher des enfants et procurer des faux papiers aux réfugiés menacés de déportation. Il constate la solidarité agissante des pasteurs et des villages protestants à l'heure où la France fait la chasse aux Juifs. Ses protecteurs du Chambon fournissent un poste d'enseignant et une pension à son maître Georges Vajda, qui s'établit ainsi à quelques kilomètres des Chouraqui. Tous les jours, les deux hommes consacrent quelques heures à travailler à leur passion commune : la Bible et Bahya Ibn Paquda. Vajda (l'un des plus grands médiévistes de ce temps, hébraïsant et arabisant distingué) prépare un livre (qui paraîtra en 1956) sur le théologien juif médiéval, chantre de l'amour de Dieu. Chouraqui termine sa traduction des Devoirs des coeurs de Bahya (qui seront préfacés par Vajda et publiés en 1950). Dans la clandestinité, Chouraqui rencontre le bibliste et cabaliste juif Jacob Gordin, l'historien Jules Isaac ainsi qu'Albert Camus, réfugiés tout près de là.

Quand Paris est libéré, les Chouraqui s'y réinstallent en octobre 1944 avant de repartir en Algérie, en 1945, où André Chouraqui sera magistrat durant dix-huit mois : juge de paix à compétences étendues. À Michelet d'abord (en Kabylie, dans le Haut-Atlas, assez proche d'Alger pour qu'il y passe son diplôme supérieur de droit musulman) puis à BouSaada, où il découvre notamment les mœurs des tribus arabes nomadisantes. Mais son épouse est malade. Les Chouraqui rentrent en France. 


\section{AU SERVICE DU PEUPLE JUIF}

À Paris, la rencontre qui fut décisive pour l'adulte de trente ans, sorti des tourments de la guerre mais cherchant encore sa voie, fut celle de René Cassin, juriste, président du Conseil d'État, principal rédacteur de la Déclaration des droits de l'Homme (et futur prix Nobel de la Paix, en 1968), mais aussi Président de l'A.I.U., l'Alliance israélite universelle. Depuis 1860 , cette institution contribue à répandre la culture juive éclairée et l'humanisme français en créant notamment un réseau serré d'écoles juives francophones d'abord à travers le Maghreb et l'ensemble du bassin méditerranéen puis dans les autres continents. Après la Shoah, elle participe à la reconstruction du judaïsme européen et à la renaissance juive dans l'État d'Israël.

En novembre 1947, René Cassin nomme André Chouraqui secrétaire général adjoint de l'A.I.U., poste qu'il occupe jusqu'en 1952. Peu fait pour les tâches administratives, Chouraqui veut démissionner. René Cassin invente alors, exprès pour lui, un poste de délégué permanent de l'Alliance qui le laisse libre de se consacrer six mois par an, dans la résidence de son choix, à son œuvre scientifique et littéraire. Délivré du souci de gagner sa vie, André Chouraqui peut désormais se consacrer à ce qui compte vraiment pour lui : la Bible, la renaissance du peuple juif d'après-guerre et la fraternité abrahamique (le rapprochement entre les trois religions issues d'Abraham).

Avec Jules Isaac et Edmond Fleg, du côté juif, les pères Daniélou et Riquet, du côté chrétien, il avait en effet participé, en 1948, à la fondation des Amitiés judéo-chrétiennes. Il ne cesse plus d'avoir des contacts avec la hiérarchie catholique, l'Église protestante et les dignitaires musulmans, créant même en 1958 un Comité pour l'entente religieuse en Israël et dans le monde, qui réunit les trois fois monothéistes (et dont il deviendra le président). À partir des années 60, une grande partie de ses essais sont consacrés à la coexistence.

Les trente années que passe André Chouraqui au côté de René Cassin, de 1947 à 1976 (date où René Cassin est mort et où Chouraqui démissionne de l'Alliance), sont parmi les plus actives et les plus fécondes de sa vie. Il poursuit sa traduction des chefsd'œuvre de son patrimoine culturel : après Bahya Ibn Paquda, il traduit et commente le poème mystique de Salomon Ibn Gabirol La Couronne du Royaume (Revue thomiste, 1952, rééd. éd. Fata Morgana, 1996) puis deux des textes bibliques qui ont marqué particulièrement l'histoire littéraire et la spiritualité : Le Cantique des Cantiques (1951) et Les Psaumes (1955). Ces traductions rencontrent immédiatement la faveur du public. Elles sont (nous l'avons vu) limpides, écrites dans un français littéraire et souple. Il ne s'agit pas encore de secouer les habitudes des traductions classiques.

Entre 1950 et 1957, l'A.I.U. envoie André Chouraqui en Israël, à de nombreuses reprises, pour y jeter les bases d'un réseau d'écoles et de comités de soutien. Sa première rencontre avec Jérusalem, en août 1950, est un éblouissement. Peu à peu mûrit en lui le souhait de s'installer en Israël. Heureusement, il peut fort bien remplir son poste de représentant permanent de l'Alliance depuis la capitale de cet État juif auquel il a consacré sa thèse de doctorat en 1948. En 1958, il passe quelques mois à apprendre à parler l'hébreu moderne à l'Oulpan Akiba, près de Netanya, avant de s'installer (définitivement) à Jérusalem. À cette époque, balbutiant, le traducteur biblique est plutôt encombré par ses connaissances livresques de l'hébreu des prophètes. En 1964, il emménage (avec sa seconde épouse) dans la maison qu'ils ont fait construire à Jérusalem, à la frontière jordanienne. Leurs cinq enfants, nés entre 1959 et 1968, achèvent de faire d'eux des Israéliens bien enracinés.

L'establishment israélien découvre, non sans surprise, cet intellectuel sépharade qui sort des salons parisiens sans avoir oublié l'arabe et sa culture maghrébine. Très vite, il est sollicité par divers milieux politiques. Il refuse de se laisser séduire, acceptant uniquement de devenir le conseiller (bénévole) du Premier ministre David Ben-Gourion en matière 
d'intégration des immigrants. Ses efforts lui valent d'être remarqué par Teddy Kollek, candidat à la mairie de Jérusalem, qui, lors des élections municipales de 1965, lui propose d'être second de sa liste. Chouraqui accepte. La victoire électorale l'installe pour deux mandats dans le fauteuil d'adjoint au maire de Jérusalem, jusqu'en décembre 1973.

Ces huit années d'action municipale sont parmi les plus concrètes de sa vie : spécialement chargé de la culture et des relations interconfessionnelles, il a aussi pour fonction d'orienter la politique urbaniste de la ville trois fois sainte, de l'équiper d'égouts modernes, d'écoles, de jardins... Il faut veiller, surtout, au fragile équilibre qui règne entre les communautés religieuses, plus encore après la guerre des Six Jours, en 1967. André Chouraqui se fait l'homme du dialogue.

La fin de son second mandat est marquée par le choc de la guerre du Kippour, en octobre $1973: 2522$ soldats morts et plusieurs milliers de blessés, côté israélien. L'auteur de la Lettre à un ami arabe (préfacée en 1969 par Shimon Pérès) est désemparé. Il aspire au silence. Il souhaite se retirer de la vie politique pour se consacrer tout entier à la traduction biblique que vient de lui commander, un an plus tôt, Jacques Deschanel. Il se retire de la mairie de Jérusalem à l'issue de son second mandat, fin 1973. En 1976, il quitte l'Alliance après la mort de René Cassin. Désormais il est indépendant, libre de se vouer à l'œuvre qu'il se sent appelé à réaliser pour couronner sa vie.

\section{TRADUIRE LES ÉCRITURES}

Dès les années 50, après le succès de ses premières traductions (Bahya, Le Cantique des Cantiques, Les Psaumes), Jacques Madaule avait demandé à André Chouraqui de s'atteler à l'ensemble de la Bible hébraïque. Il avait refusé. Il ne se sentait pas habilité à entreprendre une telle tâche. Mais lorsqu'il s'installe en Israël, l'hébreu, nous l'avons vu, devient peu à peu sa langue quotidienne et les paysages qu'il parcourt sont ceux de la Bible. «Ma lecture de la Bible en fut renouvelée, écrit-il dans son autobiographie; je comprenais mieux des textes que j'avais traduits et que je connaissais par cœur comme si un voile opaque était tombé de mes yeux» (p. 460). Il assiste régulièrement aux rencontres du fameux cercle biblique qui se tiennent, depuis décembre 1958, au domicile du Premier ministre David Ben-Gourion et qui réunissent les meilleurs spécialistes de l'exégèse et de toutes les disciplines scientifiques susceptibles d'éclairer la connaissance des Écritures. Cette nouvelle familiarité immédiate avec la Bible s'accompagne du sentiment aigu que tout ce qu'il avait vécu jusque-là le préparait à cette œuvre : l'Algérie polyglotte, la rigueur et l'élégance du style et de la réflexion «à la française», l'enseignement de l'École rabbinique, les longues périodes de méditation de la Bible et du Coran en compagnie de juifs, de chrétiens et de musulmans, ses retraites dans des séminaires ou au fond du désert saharien, enfin sa rencontre avec l'hébreu et avec les paysages où se déroule la Bible comme réalité vivante et non plus livresque. Lorsque, donc, Jacques Deschanel lui propose de traduire l'ensemble de la Bible, il n'hésite plus :«Ce fruit que je portais était parvenu à maturité : il fallait le cueillir» (p. 460).

En vingt-huit mois, du 10 avril 1972 au 14 août 1974, André Chouraqui écrit de sa main, à l'encre noire, le premier jet de sa première traduction intégrale des trois parties de la Bible hébraïque (Pentateuque, Prophètes, Hagiographes) et des quatre Évangiles (Les Quatre Annonces). Il traduit seul, en deux ans, des textes mûris en Orient sur plus d'un millénaire qui ont forgé la conscience occidentale, judéo-chrétienne, et dont il souligne l'unité profonde (notamment par l'unification du vocabulaire sémitique commun). Une fois dactylographiés (l'ordinateur personnel est inconnu à l'époque), les tapuscrits sont envoyés à des spécialistes qui révisent, annotent, critiquent. Remis sur le métier, le texte est revu par Chouraqui, renvoyé à d'autres spécialistes, retouché à nouveau. La première version de 
la Bible hébraïque et du Nouveau Testament paraît alors en 26 volumes, entre 1974 et 1977, chez Desclée de Brouwer. Le texte est publié nu, sans notes ni commentaires, découpé en volumes, en chapitres et en versets qui correspondent à l'usage juif ancestral («massorétique»). Chaque volume retrouve le titre employé dans la tradition hébraïque, c'est-à-dire le premier mot de chaque livre plutôt qu'un titre programmatique (annonçant le contenu de l'ouvrage). Le Pentateuque est à nouveau composé de cinq (penta) livres : un volume pour la Genèse (devenue: Entête, Beréchit), un pour l'Exode (Les Noms, chemoth), et ainsi de suite.

Parallèlement, il édite une Vie quotidienne des hommes de la Bible (1978) qui reprend et complète le livre paru en 1971 : La vie quotidienne des Hébreux au temps de la Bible (cf. aussi Moïse, éd. du Rocher, 1995, prix Méditerranée). Il définit son credo pour la collection de Grasset «Ce que je crois» (1979).

Devant le succès inattendu et immédiat de la «Bible Chouraqui», la preuve étant faite que le texte brut peut se suffire à lui-même (comme dans l'usage juif liturgique), Chouraqui ne considère pas que sa tâche est achevée. À ses yeux, si le texte original est immuable, la traduction ne doit cesser d'évoluer, en fonction des besoins divers, du publiccible et des nouveaux acquis des sciences et de la linguistique. Il remet son ouvrage sur le métier, harmonisant toujours plus sa restitution d'une même racine étymologique (hébraïque, arabe ou grecque) à travers son corpus, en se servant des concordances et, plus tard, des outils informatiques (suivant en cela le vieux principe d'herméneutique juif de la guezéra chava, précurseur du concept contemporain de l'«intertextualité», cf. Kaufmann, 1997). Il veille aussi à serrer chaque fois de plus près les sonorités et les significations de l'original. Parallèlement, il se résout à encadrer le texte biblique d'explications destinées à en faciliter l'accès. Le texte n'est plus livré de manière abrupte, comme un objet littéraire invitant le lecteur à une lecture suivie (ou comme un objet rituel destiné à la récitation à voix haute). Il se donne (sur la page du livre) comme un objet d'étude (dans la tradition des Mikraot Guedolot, ces éditions juives de la Bible où le texte apparaît encadré d'exégèses de toutes les époques et de toutes les écoles de pensée, invitant à une lecture «décloisonnée», bien avant que n'ait été élaborée la notion «d'hypertexte» des produits multimédias contemporains). Cette fois, André Chouraqui dispose pour l'assister d'une large équipe de consultants (biblistes, archéologues, conservateurs de musées). Le résultat est une édition augmentée de commentaires œcuméniques et illustrée de 3500 photographies d'objets ou de sites bibliques, de cartes et de graphiques, constituant une référence encyclopédique : L'Univers de la Bible (Brépols-Lidis, 1982-1985).

Pendant ce temps, il prépare, pour Desclée de Brouwer, une nouvelle édition «décloisonnée» (à usage plus «rituel») de l'ensemble de la Bible nue telle qu'elle est lue par les juifs, les catholiques et les protestants (Bible synagogale et Nouveau Testament/ Le Pacte neuf: Évangiles, Épîtres et Deutérocanoniques), qui paraît en un seul volume de 2432 pages, en 1985 (texte repris en édition de poche en 1989 et, en 1995, sur CD, collection CIB-Microbible, Maredsous).

Pour clore l'édifice, il met la dernière main à sa traduction du Coran, entamée en 1984, qui paraît chez Robert Laffont en 1990 sous le titre L'Appel après avoir été vérifiée et révisée par des arabisants et des spécialistes de l'Islam.

À partir de 1992, il entame une troisième étape de son entreprise de translateur. Il reprend, revoit et corrige l'ensemble de sa traduction des textes sacrés, publiée à nouveau sous forme de volumes individuels chez Laffont. Mais à présent, chaque volume est accompagné d'un commentaire inédit et de notes qui, pour la première fois, donnent moins les clés du texte que les clés de la traduction, expliquant notamment «les choix souvent révolutionnaires et les néologismes de la traduction. Elles en donnent les raisons, inspirées 
par la linguistique moderne ou encore par les libertés que permet l'éclatement actuel de la langue française» (introduction d'André Chouraqui à l'édition Laffont).

\section{LA MÉTHODE CHOURAQUI}

Les audaces de la Bible Chouraqui scandalisent ceux qui considèrent ses jongleries verbales comme un avatar du «littéralisme» et du mot à mot. Elles éblouissent au contraire ceux (notamment les hébraïsants) qui sont sensibles à la force poétique et créatrice de procédés propres à révéler des associations sémantiques qui auraient été perdues autrement. En jouant sur les étymologies, en utilisant les mots crus et concrets de l'original, en recourant au présent intemporel (mieux apte à rendre les temps grammaticaux hébraïques qui découpent la temporalité différemment), Chouraqui souhaite (nous l'avons vu) secouer la poussière qui couvre des siècles de routine traductionnelle, «décoloniser» le texte, le «réorienter» en le rendant (en français) à cet Orient dont il est issu, le décaper pour en retirer les mots (dont certains ont été choisis il y a vingt-deux siècles par les Septante) qui ont perdu avec le temps leur force et leur signification originelle. C'est ainsi qu'il cesse de traduire malakh par «ange» pour éviter les associations désormais inévitables entre le «messager» biblique et les angelots joufflus de l'art occidental. Il rompt la force de l'habitude en traduisant l'appel à la bien-aimée du Cantique des Cantiques (II, 10 et 14) : lekhi lakh «va vers toi-même» (l'envoyant vers la liberté) au lieu du traditionnel : «Viens-t'en» (qui est un contre-sens, un «sens» inversé, tant dans l'espace que dans la langue). Le roua'h hakodèch cesse d'être «le Saint-Esprit» pour redevenir le «souffle sacré».

De fait, comme d'autres traducteurs juifs de l'époque moderne (Martin Buber et Franz Rosenzweig en allemand, Alexandre Weil et Edmond Fleg en français), Chouraqui amène le lecteur au texte et non le texte au lecteur pour produire une sorte de Targum que Meschonnic et Gergely nommeront «une traduction-calque de la Bible». La syntaxe française est souvent malmenée pour restituer les rebonds du texte, le vocabulaire est secoué pour reconstituer les significations premières des radicaux bibliques ou pour faire écho aux sonorités hébraïques. Certains lecteurs s'en réjouissent, avides de se forger une familiarité plus grande avec une parole trop longtemps «policée» sous son vêtement français. C'est ainsi que les noms propres (de lieux, de personnages), déformés par des siècles de traduction, retrouvent leur forme première, la seule authentique, affirme Chouraqui puisque le nom, c'est l'être : Yts'hak, Ya'aqov, Essav, Moshé, Yehoshou'a, Yiov, Yrmiah (Isaac, Jacob, Esaü, Moïse, Josué, Job, Jérémie) mais aussi Yardène, Kena'an, Mitsraïm (Jourdain, Canaan, Égypte). Les personnages et les lieux des Évangiles dont les noms hébreux ont été délibérément masqués en traduction par un usage qui les a déjudaïsés retrouvent leur prononciation d'origine : Iéshou'a (Jésus), Io'hanan (Jean), Kephar-Na'houm (Capharnaüm). Mais surtout, le nom ineffable du Dieu d'Israël, oublié sous les déguisements que lui ont donnés les civilisations d'accueil, retrouve sa forme hébraïque : «Oui, je suis saint, moi-même, $Y H W H$, votre Elohim» (Il crie.../ Lévitique, XIX, 2). Les surnoms traditionnels de substitution : «le Seigneur», «l'Éternel» ou «Dieu» (vocable dérivé du latin Deus issu du grec Zeus, dieu de l'Olympe) sont écartés au profit du nom que se donne lui-même, dans l'original hébreu, Elohim : $Y H W H$, tétragramme imprononçable puisque les voyelles ne sont articulées avec les consonnes qu'une fois par an, au moment où le grand prêtre pénètre dans le Saint des Saints, dans le Temple de Jérusalem, au jour du Grand Pardon (Kippour). Dans les éditions ultérieures de sa Bible, Chouraqui invente même un logogramme qui représente simultanément la variante orale $(\mathrm{kri})$ et la représentation écrite (ktiv) du tétragramme, puisqu'il encastre et superpose la prononciation traditionnelle Adonaï (mon maître, mon Seigneur) à l'intérieur des quatre consonnes du tétragramme : 
$I H W H$ (de la racine $H W H$, le verbe «être» en hébreu). Dans son bureau d'Abou-Tor, où il me reçoit, André Chouraqui s'anime :

Ce qui restera de ma traduction : c'est que pour la première fois, Elohim a son vrai nom : qui désigne l'Être. J'ai vérifié dans les Actes d'un colloque organisé en 1982 par l'Université catholique de l'Ouest sous le titre : Dieu et Dieux, noms et Nom (Taillé, 1983). Il y a 2060 traductions de la Bible. Aucune ne porte autre chose qu'une variation sur «Theos Deos» (donc «Zeus»), même chez les Juifs. Or le décalogue dit bien : Je suis YHWH ton Elohim. Tu ne prononceras pas le nom de YHWH ton Elohim en vain (et non pas de Theos Deos). Ma traduction introduit le respect du Nom (et ce n'est pas, comme l'a écrit un critique, par souci d'exotisme). C'est la première fois depuis 2000 ans. Cela mettra des années à s'imposer, mais ce sera ma contribution.

Et Chouraqui me montre des lettres de lecteurs qui emploient spontanément la dénomination «Elohim» pour lui parler de «Dieu». De fait, Chouraqui tente d'échapper à l'éternel paradoxe du traducteur:

Chaque traducteur, plutôt que d'être attentif au message qu'il traduit, se l'accapare pour l'intégrer dans le tissu de sa propre culture et de son idéologie, sans avoir le souci d'envisager les conséquences de cet égocentrisme linguistique sur une vraie communication humaine [...] Pris entre les exigences contradictoires de la fidélité au texte et le désir d'être compris et apprécié de ses lecteurs, le traducteur est un être déchiré, souvent tenté de «faire mieux» que le texte qu'il enrobe dans une esthétique étrangère, ce qui a des conséquences incalculables quand il s'agit des textes fondateurs des grandes religions. (L'amour fort comme la mort, p. 473)

Pour sa part, Chouraqui écoute parler le texte. Il se libère de la tyrannie des dictionnaires et des habitudes de traduction. Il interroge directement la racine hébraïque ou, pour le Nouveau Testament et le Coran, la signification hébraïque qu'il sent percer, par «rétroversion», sous le vocable arabe ou le calque grec (un peu comme il retrouvait l'inspiration de l'hébreu ou le souffle de l'arabe sous le jargon français qu'employait son grand-père maternel, à Aïn Témouchent). Pour lui, comme pour d'autres chercheurs contemporains, il est flagrant que le Nouveau Testament (plus exactement Le Pacte neuf, haberith ha'hadacha: «la nouvelle alliance») a été écrit par des hommes qui - à l'exception de Luc - parlaient, comme Jésus, l'hébreu ou l'araméen et étaient nourris d'enseignements bibliques. Le Coran quant à lui (L'Appel, de la même racine que Mikra:KR', «appeler», «crier», «lire»), est écrit dans une langue sémitique, s'appuie sur un substrat proche de celui de la Bible et abonde en réminiscences et en personnages bibliques. Dans sa traduction, Chouraqui s'attache à mettre en évidence l'influence de la Bible hébraïque, de son vocabulaire et de ses images sur les textes des religions issues d'Israël. Moins pour les «judaïser» que pour en souligner l'homogénéité et dévoiler, à travers la proximité des langues et des expressions, l'unité primordiale de la parole divine, la véritable dimension œcuménique des textes. Puisque l'esprit des trois textes a la même origine, antérieure aux schismes et aux querelles qui ont séparé et divisé le judaïsme, le christianisme et l'islam, il est temps de les utiliser pour en faire de vrais ponts entre les hommes et les peuples au lieu de les tourner en armes de diffusion d'intégrismes ennemis, pense Chouraqui.

Chouraqui emploie donc, systématiquement, une même racine française pour traduire une même racine hébraïque ou sémitique, tout au long de son triple corpus, au mépris de la répétition et quelle que soit l'incorrection produite en français. Il accentue sciemment cette prescription dans la seconde puis dans la troisième version de sa traduction (1985 et 1992) et effectue des révisions rigoureuses pour faire correspondre immanquablement les mêmes vocables (ainsi, le Loth metsa'hek de Entête XIX,14, rendu par 
«dérision» en 1974, est corrigé par «rieur», par alignement sur les autres occurrences du «rire»/ts'hok dans l'ensemble de la Bible; cf. Kaufmann, 1997).

Chouraqui est, par conséquent, le seul traducteur français (à ma connaissance) à pouvoir respecter l'assonance Adam/adama (Adam/l'homme, tiré de adama/la terre). En effet, dès l'édition de 1985, il introduit un néologisme qui souligne la «dérivation» et la postériorité d'Adam/le «glébeux», dans son rapport avec adama/la glèbe, tout en essayant de traduire successivement adam par le patronyme Adam ou par le substantif «Homme» (équivalence sémantique), quand le besoin s'en fait sentir. Voici quelques exemples :

«Au glébeux, il dit : ... Honnie est la glèbe à cause de toi» (Entête III,17). «Et c'est quand le glébeux commence à se multiplier sur les faces de la glèbe, des filles leur sont enfantées. IHVH dit: "J'effacerai le glébeux que j'ai créé des faces de la glèbe, du glébeux jusqu'à la bête"»... (Entête VI,1 et 7).

Nous avons vu qu'il prolonge ce principe dans ses traductions des Évangiles et du Coran où il transporte la valeur hébraïque des racines qui, selon lui, percent sous l'araméen ou sous l'arabe, en les rendant par un même équivalent français : c'est ainsi que la racine rèch-'hèt-mème (sens concret: ré'hèm «utérus», matrice, mais aussi sens abstrait: ra'hamim «miséricorde») donne lieu à des jeux de mots et à des néologismes formés sur la même racine en français. Le El malé ra'hamim (formule habituellement traduite par: «Dieu plein de miséricorde») devient, dans la traduction biblique et évangélique de Chouraqui : un «El rempli de matrices» qui «matricie» (mera'hem), qui est «matriciel» (ra'houm). De même, dans la première sourate du Coran, (Al-Fâtihat, «l'Ouvrante» dans la terminologie chouraquienne), Allah ar-ra'hmân ar-ra'him (Dieu le «miséricordieux») est rendu par «Allah le matriciant, le matriciel». (Déjà Meschonnic (1981: 91) avait noté l'origine «utérine» du vocable exprimant la miséricorde, en hébreu et en arabe.)

Pour les Évangiles, Chouraqui établit une véritable grille de correspondances entre les vocables grecs et leurs équivalents hébraïques (comme d'autres l'ont fait, par un jeu de rétroversion, pour le grec des Septante) : c'est ainsi que Nomos redevient Tora au lieu de «loi»; Logos redevient davar, «parole»; Jésus redevient un rabbi et non un «maître» (de même qu'Allah redevient «le Rabb des univers»).

Certes, on aurait beau jeu de faire la liste des incohérences, des obscurités ou des maladresses des traductions de Chouraqui. Un critique a eu raison de dire qu'un Français serait sidéré que l'on traduise «Adieu» par «Vers Allah» en arabe, car le français ne conserve plus la conscience de l'étymologie de «adieu» (Gilliot, 1991: 614). On pourrait ajouter que si Chouraqui fait dire à Sarah : mon Adon («mon seigneur»), il ne «traduit» pas (à juste titre !) Adonaï par «messire» ou «messieurs». (Pourtant, «monsieur» vient, en français, de «mon sieur», c'est-à-dire «mon seigneur».) L'étymologie ne peut rendre compte de toutes les significations historiques d'un vocable. Et les correspondances sont parfois intraduisibles : adam/adama sont bien rendus par «glébeux»/《glèbe», mais le prénom Guershom, dans la traduction de Chouraqui, n'a plus de rapport avec son étymologie (guer/«étranger», cham/«là-bas»; cf. Touboul, 1975 : 58). Et puis, quand le texte devient incompréhensible, on peut se demander si l'ensemble de l'entreprise est justifiable (par exemple : «C'est à leurs fruits que vous les pénétrerez», Mathieu VII,16, où «pénétrer» traduit, par rétroversion, le verbe hébraïque sous-jacent : $Y D H$ qui signifie «connaître», intellectuellement et physiquement).

Enfin, le fait que des textes fondateurs, rédigés en l'espace de vingt siècles, par des hommes inspirés, d'époques et de lieux différents, en des langues différentes soient aujourd'hui traduits en un seul lieu (Jérusalem) par un seul homme (un fils d'Israël), en une seule langue (le français), à partir d'une lecture de l'hébreu (original ou restitué par le jeu d'une rétroversion) a certes une visée œcuménique, presque messianique, illustrant l'ori- 
gine abrahamique du message biblique et coranique. Mais n'existe-t-il pas un risque d'uniformisation forcée, d'unification (sollicitée par le jeu du langage) de croyances et de civilisations somme toute différentes?

Disons, pour conclure, que les traductions d'André Chouraqui sont loin de représenter un simple «calque» d'un original sémitique mal digéré en français ou plaqué de force sur une langue récalcitrante. Si elles ne laissent personne indifférent, c'est qu'il est difficile de ne pas réagir devant le souffle poétique, la verdeur, l'actualité du texte qu'il restitue dans une vitalité bondissante. Chouraqui invite à redécouvrir l'original hébreu, à revisiter les paysages de l'Israël biblique, à retrouver l'Orient originel oublié sous les siècles d'académisme linguistique occidental.

L'enfant d'Aïn Témouchent, l'étudiant parisien, le juif persécuté par les nazis, le pèlerin de la reconstruction du judaïsme d'après-guerre, ont donné naissance au patriarche juvénile de Jérusalem dont l'œuvre de traduction des Écritures est animée d'une ambition messianique qu'il a lui-même, souvent, revendiquée : établir un véritable «dialogue», une quête «dia-logos» qui mène au logos, à la parole, au texte divin, à la rencontre de tous ceux qui l'ont interprété et médité avant lui; «rapprocher les lointains» en bâtissant un pont entre les cultures, les peuples et les confessions; «rédimer Babel» en rendant à l'humanité une «langue une et une parole unifiée» (Chouraqui, E/1993 : 27-28).

\section{RÉFÉRENCES}

\section{Les textes traduits}

CHOURAQUI, André (1950a/1972) : Les devoirs du cœur de Bahya ibn Paquda, Paris, Desclée de Brouwer

CHOURAQUI, André (1950b) : Le Cantique des Cantiques, Paris, Desclée de Brouwer.

CHOURAQUI, André (1956) : Les Psaumes, Paris, Presses Universitaires de France.

CHOURAQUI, André (1974-1977) : La Bible hébraïque et le Nouveau Testament, 26 volumes, Paris, Desclée de Brouwer.

CHOURAQUI, André (1982-1985) : L'Univers de la Bible, 10 volumes, Turnhout (Belgique), Brépols, Paris, Lidis.

CHOURAQUI, André (1985) : La Bible (en un volume), Paris, Desclée de Brouwer, 2432 p.

CHOURAQUI, André (1989) : La Bible, (édition de poche en un volume), Paris, Desclée de Brouwer.

CHOURAQUI, André (1990) : Le Coran. L'appel, (traduit et commenté), Paris, Robert Laffont, 1440 p.

CHOURAQUI, André (1992-93a) : Entête, Noms, Il crie, Au désert, Paroles (nouvelle traduction du Pentateuque et commentaires), 5 volumes, Paris, J.C. Lattès.

CHOURAQUI, André (1992-1993b) : Marcos, Matyah, Iohanân, Loucas (Un Pacte neuf. Évangiles), (traduction et commentaires), 4 volumes, Paris, J.C. Lattès.

CHOURAQUI, André (1994) : Louanges (Psaumes 1 à 72), (traduction et commentaires), Paris, J.C. Lattès.

CHOURAQUI, André (1995) : La Bible sur CD, collection CIB-Microbible, Belgique, Maredsous.

CHOURAQUI, André (T/1996) : Les Psaumes/ Louanges, (traduction et commentaires), Paris, éd. du Rocher.

CHOURAQUI, André (1996-1997) : Traductions et commentaires de la Bible, (traduction en portugais de la Bible de Chouraqui), 10 volumes, Rio de Janeiro (Brésil), éd. Imago.

CHOURAQUI, André (1997) : La couronne du royaume de Salomon Ibn Gabirol, Paris, éd. Fata Morgana.

\section{Les œuvres (choix)}

CHOURAQUI, André (1960) : Cantique pour Nathanaël, poèmes, Paris, éd. Corti.

CHOURAQUI, André (1969) : Lettre à un ami arabe, Paris, Mame.

CHOURAQUI, André (1971a) : Lettre à un ami chrétien, Paris, Fayard.

CHOURAQUI, André (1971b) : La vie quotidienne des Hébreux au temps de la Bible, Paris, Hachette.

CHOURAQUI, André (1972) : La saga des juifs en Afrique du Nord, Paris, Hachette.

CHOURAQUI, André (1988a) : Jésus et Paul, fils d'Israël, Suisse, éd. du Moulin.

CHOURAQUI, André (1988b) : Mers et terres (poèmes), Paris, Euroéditeur.

CHOURAQUI, André (1989) : Aigle et palombe au survol de la mer (poèmes), éditions de l'eau.

CHOURAQUI, André (1990) : L'amour fort comme la mort - une autobiographie, Paris, Robert Laffont, 518 pp.

CHOURAQUI, André (1995a) : Mö̈se, Paris, éd. du Rocher.

CHOURAQUI, André (1995b) : Jérusalem revisitée, Paris, éd. du Rocher.

CHOURAQUI, André (1996) : Jérusalem, ville sanctuaire, Paris, éd. du Rocher. 


\section{Les études}

a) Livres et revues (sélection)

ASLANOV, Cyril (à paraître) : «La traduction de la Bible : entre retour aux sources et révolution» (étude élargie de la conférence donnée à Jérusalem, le 23 juillet 1997, au Congrès de l'Institut d'études du judaïsme oriental de l'Université hébraïque de Jérusalem, Misgav Yiroushalaïm), c. 28 p.

BAR-ASHER, Chalom (1989) : «André Chouraqui, écrivain et historien», L'Intersiècle, 2, pp. 147-155.

BOGAERT, Pierre Marie (1984) : in Dictionnaire des religions, Paris, Presses Universitaires de France.

CHOURAQUI, André (E/1974) : «Sur une traduction de la Bible», Revue thomiste, LXXIV, N.S., n 4, pp. 656659.

CHOURAQUI, André (E/1994) : Reflexionen über problematik und Methode der Übersetzung von Bibel und Koran, Tübingen, J.C. B. Mohr (Paul Siebeck), (original français dactylographié, E/1993, archives personnelles de l'auteur).

DE TRYON-MONTALEMBERT, Renée (1979) : André Chouraqui, homme de Jérusalem, Paris, éd. du Cerf, $160 \mathrm{pp}$.

DE TRYON-MONTALEMBERT, Renée (1976) : «Évangiles, les quatre annonces», Sens, n 12, décembre, pp. $19-20$.

DE VRIES, Anneke et Arian J.C. VERHEIJ (1997) : «A Portion of Slippery Stones, Wordplay in Four Twentieth-Century Translations of the Hebrew Bible», Traductio, Essays on Punning and Translation, éd. Dirk Delabastita, St Jerome Publishing (Manchester, UK) et Presses universitaires de Namur (Belgique), pp. 67-94.

DREYFUS, père F. (1975) : «André Chouraqui», Revue biblique, janvier, pp. 114-118.

ELLUL, Jacques (1977) : «La Bible, traduction d'André Chouraqui», Revue des deux mondes, novembre, pp. 507-512.

DUPUY, Bernard (1976) : «Le prestige du verbe», Les Nouveaux Cahiers, été, pp. 73-74.

GERGELY, Thomas (1980) : «La version d'André Chouraqui : une traduction-calque de la Bible», Le français moderne, $\mathrm{n}^{\circ} 4$, octobre, pp. 336-345.

GILLIOT, Claude (1991): «Coran: trois traductions récentes» Revue des sciences philosophiques et théologiques, $\mathrm{n}^{\circ} 75$, pp. 613-649.

KAHN, Jean-Georges (1974) : «Rendre à la Bible son cri», Ariel, $\mathrm{n}^{\circ} 32$.

KAUFMANN, Francine (1997) : «La Tour de Babylone; de la traduction juive des jeux de mots sur quelques noms propres hébraïques de la Genèse», Traductio, Essays on Punning and Translation, éd. Dirk Delabastita, St Jerome Publishing (Manchester, UK) et Presses universitaires de Namur (Belgique), pp. 95136.

MESCHONNIC, Henri (1970) : «Traduire la Bible aujourd'hui», Les Nouveaux Cahiers, ${ }^{\circ}$ 20, printemps, pp. 43-46.

MESCHONNIC, Henri (1974) : «Le calque dans la traduction», Les Cahiers internationaux du symbolisme, 3132, CIEPHUM, pp. 66-75.

MESCHONNIC, Henri (1978): «Le calque dans la traduction ou la Bible en décalcomanie», Poésie sans réponse (Pour la poétique V), Paris, Gallimard.

MESCHONNIC, Henri (1981) : Jona et le signifiant errant, Paris, Gallimard, 136 pp.

MORAWSKA, Madeleine (1978) : «L'art de traduire — perspectives : la Bible traduite et commentée par André Chouraqui», Sidic, XI (1), pp. 23-28.

NEHER, André (1975) : «La Bible», Revue des Études juives, CXXXIV (3-4), juil.-déc., pp. 125-128.

PAUL, André : «La Bible/ traduction de Chouraqui», Encyclopédie Universalis, pp. 368-369.

TOUBOUL, Liliane et al. (1975) : «Une version 'originale' de la Bible», Les Nouveaux Cahiers, mars, pp. 55-60.

VESCO, Jean-Luc (1974) : «Traduction nouvelle», Revue thomiste, LXXIV, N.S., n 3, pp. 474-476.

WIÉNER, Claude (1974) : «Entête», Cahiers universitaires catholiques, nº 6, juillet août, pp. 44-45.

WIÉNER, Claude (1976) : «Les Quatre annonces», Cahiers universitaires catholiques, ${ }^{\circ}{ }^{\circ}$, juillet août, pp. 4041.

b) Articles de presse (sélection)

BONHOMME, Marie-Madeleine (1977) : «Une grandiose aventure de l'esprit : la Bible traduite et présentée par André Chouraqui», Nouvelles chrétiennes d'Israël, XXVI (2), printemps, pp. 67-69.

CHAUFFIN, Yvonne (1975) : «André Chouraqui, la langue de Moïse ressuscitée», Le Pèlerin, 5 janvier, pp. 2627.

CHOURAQUI, André (E/1972) : «De la difficulté de traduire la Bible», Le Monde, 2-3 avril, p. 6.

CHOURAQUI, André (1975) : «Une traduction de la Bible», Études, octobre, pp. 447-462.

CHOURAQUI, André (E/1977) : «Traduire la Bible», Foi et Langage, n 3, avril-juin, pp. 203-209.

CHOURAQUI, André (E/1996) : «Jérusalem : trois religions pour un seul Dieu», Le Figaro, 8 août, p. 7.

COHEN, David B. (1982) : «La Bible d'André Chouraqui : un coup d'édition», Les Nouvelles littéraires, 17 au 23 mars. 
DELECLOS, P. Fabien (1983) : «Les nouveautés d'un trésor ancien», La Libre Belgique, 24 janvier.

DEROGY, Jacques (1986) : «Le sourcier de la Bible», L'Express, 11 avril, pp. 46-47.

DEROGY, Jacques (1990) : «L'itinéraire d'André Chouraqui, frontalier errant», L'événement du jeudi, 23 au 29 août.

DUQUESNE, Jacques (1991) : «Abraham, notre père à tous; entretien avec André Chouraqui», Le Point, n 957 , 21 janvier, pp. 86-90.

ELLUL, Jacques (1976) : «La Bible d'André Chouraqui», Le Monde, 6 janvier, p. 8.

FOURNIER, Jean-Noël (1991) : «De la Bible au Coran», Urbanismes et architectures, mai, pp. 40-41.

GOUR, Rachel (1993) : «André Chouraqui, Entête», Communauté nouvelle, n 67, juin-juillet, pp. 100-101.

GOURE, Claude (1989) : «Conversation avec André Chouraqui : la foi d'un juif», Panorama, pp. 20-25.

HOCINE, Karina (1993) : «La Bible revisitée», $L u, \mathrm{n}^{\circ}$ 107, décembre.

HAYOUN, Maurice-Ruben (1993a) : «La Bible et les Évangiles selon Chouraqui», L'Arche, juillet.

HAYOUN, Maurice-Ruben (1993b) : «La Bible selon Chouraqui», Le Monde, 6 août.

HAYOUN, Maurice-Ruben (1994) : «Les Psaumes revus par André Chouraqui. Sur quelques ingéniosités et bizarreries du traducteur», L'Arche, octobre, pp. 81-82.

JASSINE, David (1971) : «La Bible traduite», L'Arche, n 174-175, septembre-octobre, pp. 77-79.

KAHN, Jean-Georges (1974) : «À chacun sa Bible», Tribune juive, n 299, 22 au 28 mars, pp. 18-19.

LEFÈVRE, Gabrielle (1991) : «André Chouraqui traducteur du Coran», La Cité, Bruxelles, 7 février, pp. 70-71.

LIETTI, Anna (1990) : «L'éblouissement de Chouraqui», L'Hebdo, 6 décembre, pp. 55-56.

LURQUIN, Georges (1991) : «Le Coran/L'appel, traduit et présenté par André Chouraqui», Interface, 91/40, $1^{\text {er }}$ trimestre, 15 mars, p. 7

MESCHONNIC, Henri (1974) : «Un faux littéraire», La Quinzaine littéraire, $1^{\mathrm{er}}$ au 15 septembre.

MERZOUG, Omar (1991) : «Trois voix pour l'Unique», L'Express, 14 février, p. 118.

N'MI, Kebir (1991) : «Inimitable Coran», Le Nouvel Afrique Asie, mars, pp. $42-44$.

PESSIS PASTERNAK, Guitta (1979) : «La Bible en son sens», Question de, n 39, pp. 69-87.

RÉMOND, Alain (1985) : «Une bible explosive», Télérama, 6 novembre, pp. 147-152.

SERVANT, Jean-Christophe (1991): «Rencontre avec André Chouraqui, au carrefour des religions», Ça m'intéresse, mai, pp. 86-90.

SUFFERT, Georges (1985) : «La Bible, ce n'est plus de l'hébreu», Le Point, n 692, 23 décembre, pp. 93-98.

RAUSKY, Franklin (1994) : «Quand un juif traduit l'Évangile», Actualité juive, 6 janvier.

RIQUET, Michel (1985) : «Un homme de la Bible : André Chouraqui», La Revue des Deux Mondes, décembre, pp. 618-628.

TINCQ, Henri (1989) : «Chouraqui, le saute-frontières», Le Monde, 22 décembre, p. 18.

TINCQ, Henri (1990) : «Le Coran d'André Chouraqui», Le Monde, 5 décembre.

TINCQ, Henri (1993) : «Un entretien avec André Chouraqui», Le Monde, 13 avril.

TIRARD, Philip (1991) : «Aux racines de l'unité», Le Vif/L'express, $1^{\text {er }}$ février, p. 71.

VIALLANEIX, Paul (1990) : «J'ai traduit aussi le Coran», Réforme, nº 2 382, 8 décembre, pp. 1-2.

VAN DEN BRUWAENE, L. (1976) : «Le droit au néologisme», L'ethnie française, janvier-février, pp. 40-41.

\section{AUTRES SOURCES}

La Bible de Jérusalem (1973) : traduite en français sous la direction de l'École biblique de Jérusalem (revue et augmentée, 1993), Paris, éd. du Cerf.

KAUFMANN, Francine (1990) : «Un exemple d'approche théologique de la traduction : les jugements sur la Septante», TTR, 3(2), pp. 33-51.

KAUFMANN, Francine (à paraître) : «Le discours rabbinique sur la traduction juive de la Bible», Koiné, Anno $\mathrm{V}$, (Université d'Urbino, Italie).

LADMIRAL, Jean-René (1986) : «Sourciers et ciblistes», Revue d'esthétique, n 12, Toulouse, Privat, pp. 33-42.

TAILLÉ, Michel (1983) : Dieu et dieux, noms et Nom, Université catholique de L'Ouest, (Actes du colloque du 20/2/82) Institut de perfectionnement en langues vivantes, Angers. 